\title{
Maradona entre la tierra y el cielo*
}

\author{
Marcello SERRA \\ Universidad Carlos III
}

[...] no resultaba tan fácil olvidar que Maradona venía cometiendo desde hacía años el pecado de ser el mejor, el delito de denunciar a viva voz las cosas que el poder manda callar y el crimen de jugar con la zurda, lo cual, según el Pequeño Larousse Ilustrado, significa "con la izquierda" y también significa "al contrario de cómo se debe hacer".

Eduardo Galeano, El fútbol a sol y sombra

(Abstracts y palabras clave al final del artículo)

Enviado: 1 de mayo de 2015

Aceptado: 2 de mayo de 2015

Diego Armando Maradona. El cine le ha dedicado películas y documentales. La música popular le ha cantado himnos en los géneros más diver-

sos. Tatuada en la piel de miles de aficionados aparece la reproducción de su cara, de una jugada suya, de un autógrafo o del número que desde siempre lo ha distinguido, el diez, y que aparece también en el centro del lema con el que lo designan muchos de sus aficionados: D10S. Porque lo de Maradona es un verdadero culto.

El bar Nilo, por ejemplo, es un local tradicional justo en el centro de Nápoles. Según los usuarios de TripAdvisor el café es bueno, pero lo que lo hace distinto de cualquier otro bar napolitano es la presencia de una graciosa reliquia. Un pequeño altar de tonalidades azules muestra un "sagrado cabello milagroso" de Maradona, objeto de peregrinaciones de hinchas y apasionados del fútbol, exhibido junto a un frasquito supuestamente lleno de lágrimas vertidas por los napolitanos en el momento de su despedida.

Si luego nos desplazamos a Argentina, tierra originaria del inolvidable campeón, la figura del Diego se encuentra en el centro de prácticas idolátricas aún más evidentes ${ }^{1}$. Entre todas, la más significativa es probablemente la de la religión paródica representada por la Iglesia Maradoniana, cuyos adeptos llaman a sus hijos Diego, celebran una "Navidad Maradoniana" el 30 de octubre, una "Pascua" el 22 de junio y pueden incluso contraer matrimonio según rito propio ${ }^{2}$.

\footnotetext{
* Este artículo se enmarca dentro del Proyecto I+D "El periodista como historiador del presente: análisis del documento en las nuevas formas de la información" (Ref.: CSO2014-55527-P)

1 El fenómeno de la idolatría maradoniana está bien recreado en la película de Carlos Sorín "El camino de San Diego". La historia trata del peregrinaje de Tati, un joven trabajador de la madera de la provincia argentina de Misiones. Un día, andando por la selva, Tati se encuentra con una raíz que le parece reproducir los rasgos de Maradona. Cuando llega a saber que este está ingresado en condiciones críticas en un hospital de Buenos Aires, decide ponerse en camino para entregar la estatua a su ídolo.

2 El 30 de octubre es el día de nacimiento de Maradona, el 22 de junio recuerda el del famoso partido entre Argentina e Inglaterra en el 1986, del que hablaremos más adelante. En internet es posible ver también un vídeo del primer casamiento maradoniano. Los novios se llaman Jaquelina y Mauricio, la ceremonia tiene lugar en una cancha de fútbol y el oficiante es uno de los fundadores de la Iglesia Maradoniana, cuyas palabras resultan parcialmente acalladas por el ruido de fondo de un cortacésped.
} 
Por mucho que sean generalmente irónicas, estas prácticas indican en cualquier caso la presencia de una gran fuerza simbólica que resiste al tiempo y pide una explicación. Para los fundadores de la Iglesia Maradoniana es todo muy lógico: "para los argentinos el fútbol es una religión, cada religión tiene su dios, bueno el dios del fútbol es Diego".

Las siguientes páginas serán dedicadas a intentar ilustrar el motivo de tal afirmación. Trataré de reconstruir sus razones y de mostrar cómo, más allá de su naturaleza hiperbólica, esta tiene un fondo metafórico de verdad, un fondo que reposa en la eficacia simbólica de un discurso sobre el fútbol y las gestas de Maradona.

Como cuenta el antropólogo Eduardo Archetti (2003), en la Argentina de los años veinte y treinta el arte, el cine, el tango y una serie de otras formas culturales participaron en la construcción de un carácter específico nacional, de una "argentinidad" hasta entonces desconocida. En este proceso tuvo un papel no marginal también el fútbol, en el que ya en ese tiempo se condensaban grandes pasiones populares. En las canchas se afirmaba en esos años el así llamado "estilo criollo", que revistas populares como "El Gráfico" celebraban como distintivo del pueblo argentino ${ }^{3}$. La razón de esta elección es que sus características resultaban fácilmente oponibles a las del juego británico, un hecho que resultaba importante desde el punto de vista identitario; por un lado el fútbol había llegado a Argentina junto con la Union Jack y, por otro, la relación entre los dos países era muy estrecha también en ámbito cultural y económico, hasta el punto de conformar una especie de vínculo colonial. El imaginario y las prácticas culturales relacionadas con el Reino Unido, por tanto, se ofrecían naturalmente como polo negativo sobre el que construir, por contraste, una identidad nacional argentina.

En el fútbol, el estilo británico se enseñaba en las escuelas privadas y en los clubes deportivos, caracterizándose por un acentuado atletismo y una gran atención a la organización del juego, prerrogativas que la prensa argentina relacionaba con el industrialismo y la modernidad. Al contrario, el estilo criollo se asociaba a la imagen del pibe, del muchacho crecido futbolísticamente en los potreros. Estos eran, y siguen siendo, canchitas de barrio polvorientas que reciben su nombre de aquellos terrenos baldíos de la pampa donde los caballos y el ganado podían apacentar libremente bajo la protección del gaucho, y que se veían como un territorio todavía libre, no ocupado permanentemente por la agricultura y, por tanto, como metáfora y recuerdo de la pampa salvaje.

En estos potreros de ciudad el pibe aprende sin maestros, adquiere la viveza, la astucia, y se expresa principalmente a través del regate, gesto que, por un lado, representa más que cualquier otro la maña y la individualidad futbolística, mientras que por

3 A partir de 1928, en las páginas de El Gráfico se desarrolló una teoría que postulaba dos fundaciones distintas del fútbol argentino. Con la primera se hacía referencia a la británica; la segunda tenía su origen con las hazañas del Racing Club de Avellaneda, primer club compuesto exclusivamente por jugadores locales y siete veces consecutivamente campeón nacional entre 1913 y 1919.

4 He aquí un ejemplo significativo de la posición de la prensa popular argentina sacado de un número de El Gráfico de 1928: 
otro es figura de una actitud típicamente criolla, de una habilidad "táctica" de renegociar constantemente los términos de la interacción (Jacoviello, Sbriccoli 2012).

En la visión de los periodistas argentinos de esos años, y en particular en la del famoso Borocotó, el fútbol británico era comparado a una máquina, a una actividad industrialmente perfecta que no dejaba espacio a la improvisación; el criollo, al contrario, era "inquieto, individualista, menos disciplinado, basado en el esfuerzo personal, ágil y virtuoso" (Archetti 1998: 265) . Un producto, en fin, de los pibes del potrero, un lugar que, por su parte, revela una especial densidad semiótica. Por un lado se presenta como el espacio de adquisición de una competencia futbolística ${ }^{5}$ ejemplarmente argentina por sus reenvíos al gaucho y a la pampa. Por el otro es un lugar que no solo goza de la libertad creativa típica de las periferias de la semiosfera (Lotman 1996), sino que es donde se activa un movimiento de redefinición de dinámicas "centrales", es decir, de la manera de jugar al fútbol y de la manera de entenderlo: de actividad típica de los privilegiados inmigrantes británicos a expresión del pueblo argentino y de sus clases populares.

En esta visión, el fútbol criollo resulta por tanto estrechamente asociado con la espontaneidad y la libertad de la infancia. Tanto que, en Argentina, los grandes jugadores siguen siendo pibes también cuando crecen; en el fondo no cambian y sus jugadas, las mejores jugadas, no son sino la actualización de la competencia adquirida en el potrero, de una habilidad no contaminada por el saber táctico del juego "adulto". Entre todos, Maradona es el "pibe de oro", el jugador que ha encarnado en la manera más perfecta (en la cancha y fuera de la cancha) esta genial inmadurez: de extracción social humilde, ha crecido futbolísticamente en los potreros de Villa Fiorito (Buenos Aires) y no ha reconocido nunca haber aprendido algo de un entrenador, era un as del regate, un campeón de astucia y un jugador rigurosamente impredecible. Perfectamente "pibe", y hasta hombre inmaduro.

[...] es lógico que con el correr de los años, toda la influencia sajona del football haya ido desapareciendo para dar paso al espíritu menos flemático y más inquieto del latino [...]. Inspirados en la misma escuela que los británicos, bien pronto los latinos fueron modificando la ciencia del juego e hicieron una propia, hoy ampliamente reconocida [...] ella se diferencia de la inglesa en que es menos monocorde, menos disciplinada y metódica, pues no sacrifica el individualismo en homenaje a la suma colectiva de los valores. En el football inglés todo tiende a destruir la acción personal para formar un todo sólido, de manera que un team no se cuenta por sus hombres separadamente, sino por la acción uniforme de todo un conjunto. De ahí que el football británico sea realmente poderoso y tenga la fuerza regular e impulsiva de una verdadera máquina, pero es monótono porque siempre es igual y uniforme. El football rioplatense, en cambio, no sacrifica enteramente la acción personal y utiliza más el dribbling, el esfuerzo personal generoso, tanto en los hombres de ataque como de defensa (cit. en Archetti 1998: 265).

${ }^{5}$ En términos de la semiótica generativa de Algirdas J. Greimas se trata de un espacio que se define como "paratópico". En el Diccionario razonado de la teoría del lenguaje se describe en los siguientes términos: "subcomponente del espacio tópico y opuesto al espacio utópico (en que se desarrollan las performances) es aquel en que se desarrollan las pruebas preparatorias o calificantes y se adquieren las competencias (tanto en la dimensión pragmática como en la dimensión cognoscitiva)" (Graimas, Courtés 1982: 300-301).

6 "Dios cojo" o "monosandalia" era uno de los epítetos del dios griego Dionisos, a menudo representado con un pie descalzo. 
Con estas premisas, no hay quien niegue que Maradona representa un perfecto prototipo del fútbol criollo, por lo menos si entendemos el prototipo como "el punto de mayor concentración de las propiedades típicas, el ejemplar que tiene en común el mayor número de propiedades con los demás miembros de la categoría, y al mismo tiempo el menor número con ejemplares pertenecientes a categorías distintas" (Violi 1997: 185). Por otra parte, razonando un poco más a fondo sobre la segunda parte de la definición, las características de Maradona que saltan a la vista son el físico poco vigoroso, la poquísima propensión al entrenamiento, los problemas con la báscula o la drogadicción. Y lo que es interesante es que se trata de propiedades que no se encuentran casi nunca en deportistas de alto nivel (a excepción de las disciplinas a baja intensidad agonística), pero no son tan raras en los futbolistas, como lo demuestran, entre otros, los casos de campeones como George Best o Paul Gascoigne.

En otras palabras, la prototipicidad maradoniana trasciende la dimensión argentina y se liga más en general al fenómeno del fútbol, deporte absolutamente atípico en el que se puede perder un encuentro teniendo incluso todas las estadísticas a favor. Todas, obviamente, menos la de los goles realizados. Y ello porque el gol no pertenece al normal desarrollo de las cosas, no es el éxito natural de una correcta acción de ataque. Fruto más bien de un error o de una proeza, el gol es probablemente, entre todos los puntos posibles en todos los deportes contemporáneos, el de mayor valor informativo, el más imprevisible.

No parece entonces insensato postular precisamente la imprevisibilidad como prerrogativa fundamental del fútbol. $\mathrm{O}$, por lo menos, de cierto tipo de fútbol, porque es verdad que no es propia de todos los estilos de juego ni siquiera de todos los campeones.

No era tan imprevisible, por ejemplo, el otro gran icono del fútbol mundial, Pelé, cuyas características son bajo muchos puntos de vista opuestas a las de Maradona. Dotado de un óptimo físico y velocidad, mucha fuerza y un repertorio técnico completo, Pelé ha sido un campeón apolíneo, de los que sobresalen, siguiendo la descripción de Yuri M. Lotman (2013), por poseer cualidades comunes, pero en forma cuantitativamente hipertrófica. Pertenece por tanto a la tradición de los Aquiles, de quien sigue las reglas y sobresale operando dentro de los límites definidos por ellas. Tan hábil y fuerte hasta el punto de resultar, sin embargo, casi previsible y marcar su milésimo gol de penalti.

Maradona representa, al contrario, un modelo dionisíaco y, como Dionisos, de alguna forma era "cojo"6. Utilizaba solo el pie izquierdo, dotado por otra parte de movilidad reducida por culpa de una grave lesión padecida al tobillo, y lo hacía "de zurda", recurriendo a la métis, a esa inteligencia astuta que permite atrapar al kairós (Detienne, Vernant 1988) ${ }^{7}$.

7 Entre otras cosas, esto explica también las razones por las que, además que en Argentina, Maradona es un ídolo absoluto también en Nápoles, ciudad en la que no solo ha conquistado títulos importantes, sino también donde la viveza es un valor tenido en altísima consideración.

8 "Cuando pienso en Inglaterra, no puedo sacarme de la cabeza a los pibes que murieron en la guerra de las Malvinas", había declarado Maradona días antes de aquel encuentro. 
Si Pelé detenta el récord de campeonatos mundiales ganados (tres) y de goles marcados durante una carrera (1281, pero contando también los partidos no oficiales), Maradona no posee ninguno. Y sin embargo, como afirma Hans Gumbrecht en su Elogio de la belleza atlética: "los récords, por definición, pertenecen a aquello que es comparativamente grande y, por lo tanto, no absolutamente grande". Por otra parte, añade, "todos los fanáticos del deporte tienen recuerdos de hazañas y eventos que, según lo que piensan, nunca se podrán igualar" (Gumbrecht 2006: 48). Para celebrar a otro gran campeón, Roberto Baggio, el periodista Michael Farber escribió en Sports Illustrated que "su leyenda no se ha construido sobre títulos, sino sobre momentos", no sobre la continuidad del récord, sino sobre la discontinuidad de lo memorable. Y en el mismo sentido podemos decir que la grandeza de Maradona depende de gestos incomparablemente grandes, hazañas que, en algunos casos, exceden la dimensión deportiva y devienen fuertemente simbólicas.

Entre todas, dos son las que recuerda cualquier aficionado, dos episodios acontecidos a cinco minutos de distancia uno del otro, el 22 de junio de 1986 en el estadio Azteca de Ciudad de México, en un partido de los cuartos de final del mundial de fútbol. Un partido contra Inglaterra, esa misma de la que el fútbol criollo se había querido distinguir y contra quien Argentina había perdido hace poco una guerra por la posesión de las islas Malvinas ${ }^{8}$. Un encuentro que replicaba otros cuartos de final de mundial, ganados por los ingleses entre grandes polémicas exactamente veinte años antes ${ }^{9}$.

A partir de estas premisas parece posible poner en juego, aunque descontextualizada, una famosa categoría luckácsiana; el fútbol y, en concreto, las dos famosas jugadas de Maradona devienen formas (Luckács 1916): composiciones de disonancias fundamentales de la existencia, soluciones en el plano simbólico de un conflicto irresoluble, aquel entre la ambición argentina y una realidad hecha de subalternidad política, económica, cultural y marcada, finalmente, por la derrota militar.

El primer episodio acontece al minuto 51, con el marcador a cero, cuando, con Argentina en ataque, un balón es colgado por el defensor inglés Steve Hodge y se dirige hacia el centro del área. Peter Shilton, portero de $1,86 \mathrm{~m}$ salta y, con un brazo levantado, intenta despejar el esférico, que le es disputado por Maradona, $20 \mathrm{~cm}$ más pequeño. A primera vista la acción no se presenta realmente peligrosa, ya que el resultado del duelo parece descontado a favor del último defensor inglés; un instan-

9 El partido del 1966 es famoso por el episodio de la expulsión del capitán del equipo argentino Antonio Ubaldo Rattín, justificada posteriormente por el árbitro alemán Rudolf Kreitlein con la afirmación que este le había "mirado mal" mientras protestaba. La cuestión es que Rattín no hablaba ni inglés ni alemán, y Kreitlein no conocía el español, pudiendo solo imaginar qué le había dicho el capitán argentino. La situación de incomprensión duró unos largos diez minutos, en los que Rattín se negó a dejar la cancha. Lo que pasó después está envuelto en leyenda, pero la versión aceptada por la cultura popular se resume en esta declaración atribuida al mismo Rattín: "Me senté en la alfombra roja que le pusieron a la reina. Iba a los vestuarios y me empezaron a tirar chocolatines. Cuando estaba por llegar al banderín del córner agarré una bandera inglesa que flameaba en el palito. La retorcí toda y la mostré. Entonces, en vez de chocolatines me tiraron latas de cerveza"

(http://espndeportes.espn.go.com/news/story?id=1845821\&s=futbol/mundiales\&type=story).

10 El nombre del fotógrafo era Alejandro Ojeda Carbajal, o por lo menos así parece, porque la autoría de la imagen es reivindicada también por el reportero inglés Robert Thomas. El misterio crece si consideramos 
te después, sin embargo, entre la incredulidad general la pelota entra en la red. Maradona festeja mientras en el campo y en las gradas están todos como bloqueados. Algunos ingleses protestan, pero solo el replay televisivo revela el misterio: camuflada entre la melena desordenada y un movimiento de la cabeza, es la mano de Maradona la que golpea el balón. No obstante, pocos se han dado cuenta, y entre ellos no está el árbitro, quien se dirige al centro de la cancha otorgando el tanto.

En esta jugada, tan impredecible que solo un fotógrafo entre los presentes en los bordes del campo consigue capturar el momento y fijarlo en la película ${ }^{10}$, Franciscu Sedda reconoce un momento de explosión en el sentido lotmaniano, una "conjunción de lo inconjuntable" que se presenta como "loca y desregulada inspiración, como gesto arbitrario y ofensivo de la razón común, del sentido tradicional, dado y establecido" (Sedda 2010: 294). La jugada es, por otra parte, fuertemente evocativa: un argentino no puede no ver en ella la obra del pibe del potrero y, más en general, esta trae a la mente el débil que se la lía al fuerte, el trickster, el pícaro divino que "vence con la inventiva, la rapidez de ingenio, la astucia y el engaño, en último análisis, con la inmoralidad" (Lotman 2013: 62).

Pero cuidado con los adjetivos, porque el fútbol es un deporte en varios aspectos indiferente hacia la justicia, la verdad y la moralidad misma. Ya hemos dicho que se puede ganar jugando muy mal o por un simple regalo de la suerte, añadimos ahora que se trata de un deporte con reglas a menudo elásticas: ciertas faltas se pueden conceder o se puede dejar jugar, ciertos penaltis se pitan o no se pitan sin que una de las dos decisiones sea la correcta en sentido absoluto; el árbitro interpreta, sin el auxilio de ayudas tecnológicas, según el propio metro de juicio y siguiendo su propio estilo ${ }^{11}$. Es así que la astucia y cierta indiferencia a la moral entran a hacer parte del espíritu del juego, mientras que el fair play realmente mucho menos. Como afirma un viejo dicho británico, si el "rugby is a thug's game played by gentleman, football is a gentleman's game played by thugs", donde "thug" significa bruto, pero también rufián.

No resulta por tanto sorprendente que la clase trabajadora argentina fuera de alguna manera legitimada a leer el gol con la mano como una forma necesaria de picardía criolla, especialmente si consideramos los que son los valores del potrero: "donde el uso gaucho del engaño para la supervivencia es fuente de orgullo para el nacionalismo criollo, del mismo modo, el fútbol en el potrero es coherente con ganar a toda costa incluso cuando requiere la obtención de una ingeniosa, o 'injusta', ven-

que Thomas resulta ser autor también de una segunda foto, tomada en el mismo instante pero desde la parte opuesta del campo.

11 En este sentido, la resistencia de la FIFA (federación Internacional de Fútbol Asociación) a la introducción de la tecnología en la cancha como auxilio al árbitro parece actuar como protección del espíritu "laxo" de las reglas futbolísticas. Por otra parte, como observa Hibai López (2012), la imprecisión del reglamento se halla también en la duración de los partidos, en los que el tiempo transcurrido no coincide con el jugado, y en la delimitación del campo, definido por líneas que forman parte del terreno de juego y que se proyectan verticalmente sin ningún límite. Estas características, además, hacen que tanto los confines espaciales como los temporales sean "habitables".

12 Con el término "barrilete" en Argentina se suele indicar lo que es en castellano normalmente conocido como "cometa" 
taja sobre el oponente" (Sibaja, Parrish 2014: 664). Sea como sea, Víctor Hugo Morales, uruguayo de nacimiento, pero cronista en aquel entonces para la radio argentina no tiene dudas: "que Dios me perdone lo que estoy por decir", declara en directo, "contra Inglaterra hoy, aun así, con un gol con la mano, ¿qué quiere que le diga?".

El juicio es, obviamente, partidario, y seguramente no compartido por todos los aficionados, especialmente los de la parte inglesa. Para poderse expresar al respecto, no obstante, hay que insertar el episodio en una narración más amplia, que no solo active elementos de la memoria argentina como los de la guerra de las Malvinas, sino que incluya por lo menos otro momento clave del partido, acontecido apenas cinco minutos después.

En el minuto 56 Maradona toma el balón en mitad de la cancha, se libra de cuatro jugadores, regatea también al portero y marca el que será luego universalmente reconocido como "el gol del siglo". En las palabras del periodista Brian Glanville fue "tan inusual, casi romántico, que pudo haber sido marcado por un niño héroe, o algún remoto corintio, en los días en que el regate era la moda. Difícilmente se adscribía a una época como la nuestra tan aparentemente racional y racionalizada, a un periodo del fútbol en que el regateador parecía tan extinto casi como el pterodáctilo" (cit. en Burns 2010: 160).

Glanville describe brillantemente las cualidades criollas del gol de Maradona: la creatividad "infantil", el individualismo y su carácter lúdico, más jocoso que deportivo. En este gol hay, sin embargo, todavía algo más. Para entenderlo, veamos cómo lo vivieron los argentinos a través de las palabras de Víctor Hugo Morales, locutor de radio con un nombre que condena a la poesía:

La va a tocar para Diego, ahí la tiene Maradona, lo marcan dos, pisa la pelota Maradona, arranca por la derecha el genio del fútbol mundial, y deja el tercero y va a tocar para Burruchaga... ¡Siempre Maradona! ¡Genio! ¡Genio! ¡Genio! ta-ta-ta-ta-ta-ta-ta... Goooooool... Gooooool... ¡Quiero llorar! ¡Dios Santo, viva el fútbol! ¡Golaaaaaaazooooooo! ¡Diegooooooool! ¡Maradona! Es para llorar, perdónenme... Maradona, en recorrida memorable, en la jugada de todos los tiempos... barrilete cósmico... ¿de qué planeta viniste? ¡Para dejar en el camino a tanto inglés! ¡Para que el país sea un puño apretado, gritando por Argentina!... Argentina 2 - Inglaterra 0... Diegol, Diegol, Diego Armando Maradona... Gracias Dios, por el fútbol, por Maradona, por estas lágrimas, por este Argentina 2 - Inglaterra 0.

Entre todas las crónicas de este episodio en lengua española, esta es seguramente la más famosa, aparece en la entrada "gol del siglo" de Wikipedia y acompaña las reproducciones más populares en YouTube. Es por esta razón que me parece oportuno considerarlo como un elemento importante en la construcción del punto de vista argentino tanto sobre el valor simbólico del acontecimiento como sobre la figura de Maradona.

Procediendo por orden, el primer elemento que se puede subrayar es que Morales llama a Maradona "genio", un epíteto que en el discurso en cuestión adquiere distintos matices. Siguiendo el diccionario de la Real Academia Española podemos observar antes de nada que indica alguien con una "capacidad mental extraordinaria para crear o inventar cosas nuevas y admirables", características que Maradona había demostrado en varias ocasiones y confirmado pocos minutos antes, paralizan- 
do el estadio con un gol con la mano; en segundo lugar remite a la idea del genio de la lámpara (explícitamente citado como ejemplo en la definición de la RAE), una entidad que concede deseos y que parece ser evocado en la triple repetición inmediatamente sucesiva: “¡Genio! ¡Genio! ¡Genio!”, que acompaña la entrada en el área de penalti; finalmente, "genio del fútbol" reenvía a la "condición peculiar de algunas cosas", indicando la propiedad de encarnar las cualidades mismas del fútbol, de ser un prototipo.

Inmediatamente sucesiva a la triple invocación del "genio", el "ta-ta-ta-ta-ta-ta-ta" que coincide con el regate al portero y el tiro es, por su parte, una expresión de la inefabilidad del gesto, que Sedda asimila a una turbulencia, a "una puesta en movimiento de todos los elementos del sistema, sobre todo de aquellas posibilidades tan latentes que resultan inopinadas o aparecen imposibles" (Sedda 2010: 300). Su manifestación "nos enciende, nos acalora, hace evaporar todo buen sentido para dejar espacio a un eufórico turbamiento" (Ibidem) y, de hecho, Morales exclama que quiere llorar. Luego invoca a Dios y celebra al fútbol, del que Maradona deviene la encarnación: "Diegol", es decir, el fútbol en su imprevisibilidad.

En este punto, todo se hace absoluto. Morales advierte que la "recorrida" realizada por Maradona es "memorable" y que se trata de la "jugada de todos los tiempos". Lo afirma porque ha sido testigo de ella, al contrario que sus oyentes él ha visto y siente poder sancionar el valor histórico del acontecimiento ${ }^{12}$. De un hecho, sin embargo, que no es de esta tierra. En las palabras de Morales, Maradona deviene un "barrilete12", un ser ligero, impredecible y que, como el Dios cristiano, pertenece a un cielo remoto, "cósmico". Esta lejanía desmesurada es confirmada por la pregunta sucesiva, que arroja a Maradona de una vez para siempre a un espacio y a un tiempo inaccesibles, originarios, míticos: "¿de qué planeta viniste?", pregunta Morales, introduciendo de improviso un pretérito que recuerda al perfecto épico descrito por Käte Hamburger (2009), un tiempo que no indica el pasado sino la ficción, la irrealidad del cuento. La pregunta es un "como si" que señala las consecuencias fuertemente simbólicas del acontecimiento: el país es ahora "un puño apretado gritando por Argentina", el gesto de júbilo de todo hincha se ha transformado metonímicamente en la condición de una patria unida y vencedora gracias a Maradona ${ }^{13}$. Este, por su parte, deviene una entidad casi divina; Morales lo deja claro con su última frase, en la que agradece a Dios por el fútbol y por Maradona. Y puesto que, anteriormente, entre Maradona y el fútbol se había establecido una equivalencia, la isotopía religiosa del relato radiofónico hace reconocer de inmediato una analogía con la trinidad cristiana: Maradona es un dios bajado a la tierra a través de la mediación del fútbol.

La narración de Morales, que podríamos casi incluir en el género de la eulogía ${ }^{14}$, construye admirablemente la actitud que hay que tener hacia el acontecimiento: los

13 Sobre el periodista como historiador del presente cfr. Lozano 2013. Sobre el rol del testigo en la escritura de la historia cfr. Lozano 1987, Wieworka 1998 y Hartog 2007.

14 Además, Morales está describiendo una situación de abandono dionisíaco que es típica de las masas de hinchas. Y la posibilidad que esta intoxicación de felicidad sea extendida al territorio nacional argentino es concedida por la cualidad mediática del acontecimiento, de las imágenes de la televisión y del mismo relato radiofónico de Víctor Hugo Morales, que implican y transforman al público de forma visceral, no solo en el nivel cognitivo, sino también en el pasional o somático.

15 Agradezco a Stefano Jacoviello por esta sugerencia. 
argentinos deben festejar, conmoverse y sentirse vengados. En términos narrativos greimasianos, deben también entender que el gol del siglo es una prueba glorificante que lo hace reconocer como la encarnación del fútbol y que sanciona su poder ser más allá de lo límites concedidos a los jugadores normales.

Vendría la tentación de decir más allá del bien y del mal, y no sería del todo incorrecto. En este caso Maradona infringe la norma, va más allá de lo que se pensaba posible. Cinco minutos antes había roto las reglas, pero es esta una transgresión resemantizada por la siguiente, que hace de Maradona un héroe, una figura "a mitad de camino entre los dioses y los mortales" (Morin 1964: 44) ${ }^{15}$. Un semidiós del que el segundo gol representa la mitad celeste y el primero la terrenal. Por esto al final del partido, a comentario del primero, podrá declarar que había sido "un poco con la cabeza y un poco con la mano de Dios", en un acto, como bien observa Sedda, de delegación del gesto "a una entidad sacra, un destinador que irrumpe rompiendo las reglas para un bien y una justicia superior" (Sedda 2010: 294). En la terminología de Richard Rorty podríamos decir que los cinco minutos del estadio Azteca fueron la obra de un "strong poet" que redefinió los límites de las normas futbolísticas (Loland 2005). Lo que estaba prohibido pasó a ser admisible. Y así Jorge Valdano, que ese día estaba en la cancha, pudo declarar que "en Argentina el gol malo fue más festejado que el bueno, porque fue contra los ingleses, y parecían merecerlo" (Britto et al. 2014: 683).

Entre trozos de memoria, relatos periodísticos y declaraciones de prensa se configura así un discurso perfectamente eficaz: el brinco con el que Maradona anticipa a Shilton, ese instante inmortalizado en una foto única, ha pasado a la historia no como el signo de una trampa, sino como la traza de un héroe suspendido en vuelo, de un mediador entre la tierra y el cielo.

16 El pasaje sigue así: "[los héroes] ambicionan alcanzar la condición de los dioses y aspiran a librar a los mortales de su miseria infinita. En la vanguardia del hombre, el héroe es el mortal en proceso de divinización. Emparentado con los dioses y con los hombres, los héroes de los mitos, con mucha justicia, se llaman semidioses" (Morin 1963: 44-45). 


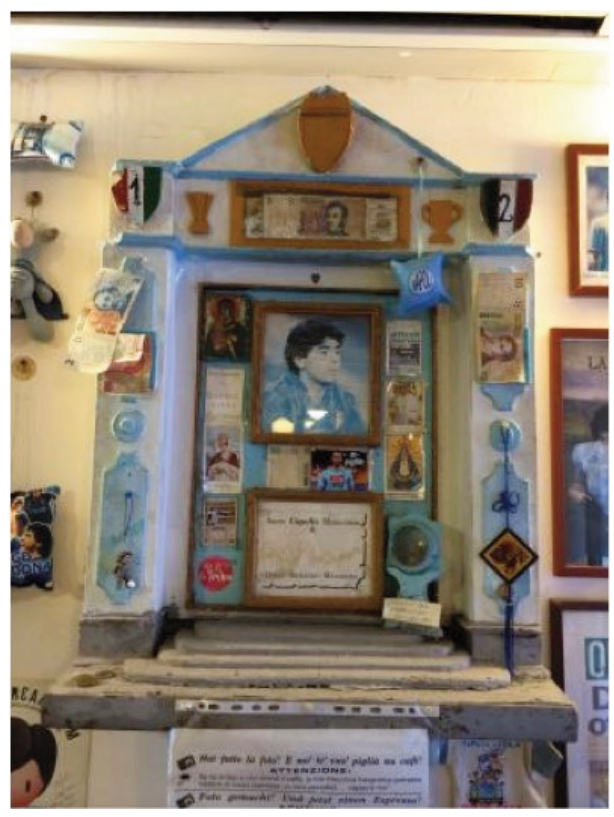

Altar de Maradona en Nápoles.

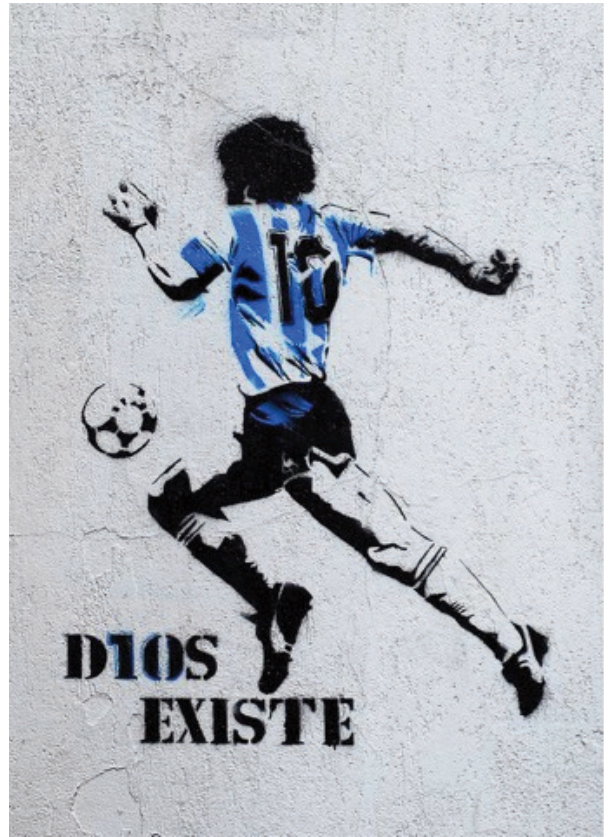

Estarcido realizado a partir de la imagen de una jugada de Maradona. 


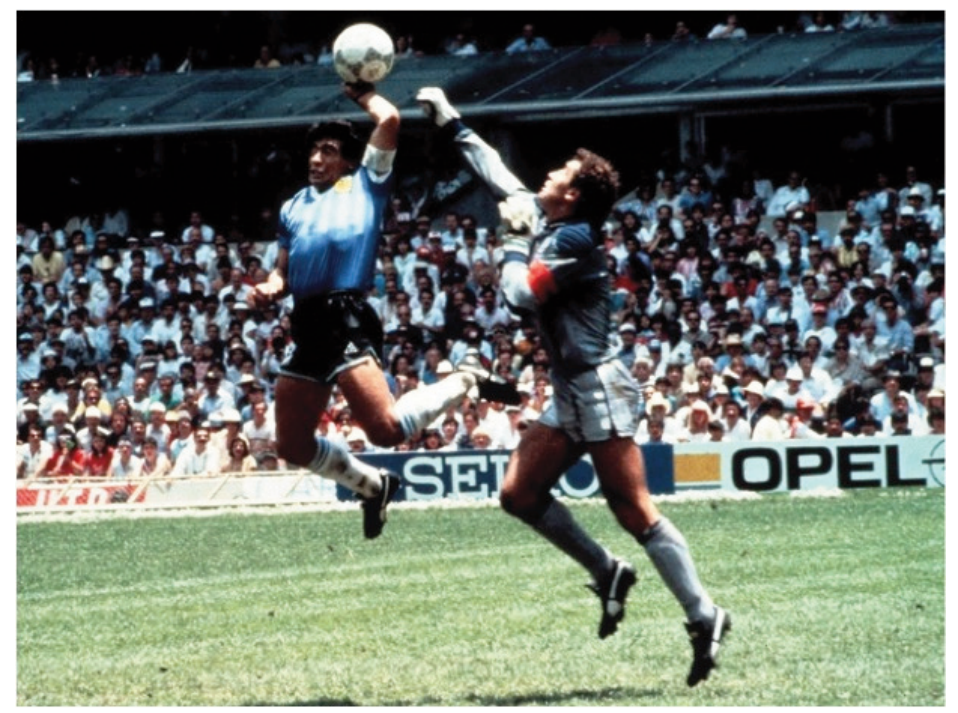

La "mano de Dios".

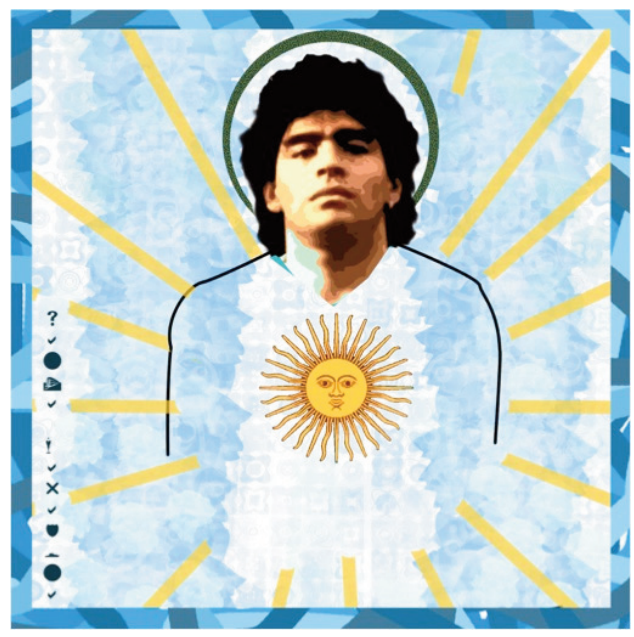

Fondo de escritorio de Maradona como santo 


\section{BIBLIOGRAFÍA}

ARChETTI, Eduardo P. (1998), "El potrero y el pibe. Territorio y pertenencia en el imaginario del fútbol argentino" en Nueva Sociedad, 154, pp. 101-119.

- (2001), "The spectacle of a heroic life: the case of Diego Maradona", en ANDrews D. L., JACKSON S. J., eds., (2001), Sport Stars. The cultural politics of sporting celebrities, Routledge, London /New York, pp. 151-163.

- (2003), "Playing Football and Dancing Tango: Embodying Argentina in Movement, Style and Identity", en Dyck N., ArchetTi E. P., eds., (2003), Sport, Dance and Embodied Identities, Berg, Oxford, New York, pp. 217-229.

Britto, Simone; Morais, Jorge; Barreto, Tulio (2011), "The Hand of God, the Hand of the Devil: a sociological interpretation of Maradona's hand goal”, en Soccer \& Society, Vol. 15, No. 5, 671-684.

BuRns, Jimmy (2010), Maradona: The Hand of God, Bloomsbury, London.

Cervelli, Pierluigi; Romei, Leonardo; Sedda, Franciscu (2010), Mitologie dello sport. 40 saggi brevi, Edizioni Nuova Cultura, Roma.

Detienne, Marcel; Vernant, Jean Pierre (1988), Las artimañas de la inteligencia, Taurus, Madrid.

Greimas, Algirdas J.; Coutés, Joseph (1982), Semiótica. Diccionario razonado de la teoría del lenguaje, Gredos, Madrid.

Gumbrecht, Hans (2006), Elogio de la belleza estética, Katz Editores, Buenos Aires.

HAMBURGer, Käte (2009), "La finzione narrativa”, en Allegoria, N. 60, pp. 13-41.

HARTOG, François. (2007), Evidencia de la historia. Lo que ven los historiadores, Universidad Iberoamericana, México.

Loland, Sigmund (2005), "The Varieties of Cheating-Comments on Ethical Analyses in Sport", en Sport in Society: Cultures, Commerce, Media, Politics, 8:1, pp. 11-26.

Lotman, Yuri M. (1993), Cultura y explosión. Lo previsible y lo imprevisible en los procesos de cambio social, Gedisa, Barcelona 2013.

Lotman, Iuri M. (1996), La semiosfera I, Cátedra, Madrid.

LozAno, Jorge (1987), El discurso histórico, Alianza, Madrid.

LozANo, Jorge (2013), “El discurso periodístico: entre el discurso histórico y la 'fiction'. Hacia una semiótica del acontecimiento", en Estudios sobre el Mensaje Periodístico, Vol. 19, No 1, pp. 165-176.

LUCKÁCS, György (1920), Teoría de la novela, Edhasa, Barcelona 1971.

MorIn, Edgar (1964), Las estrellas de cine, Editorial Universitaria de Buenos Aires, Buenos Aires.

SEDDA, Franciscu (2010), "Maradona e l'esplosione. Dalla Mano di Dio al Poema di gol", en

Cervelli, P.; RomeI, L.; SedDA, F. (2010), Mitologie dello sport. 40 saggi brevi, Edizioni Nuova Cultura, Roma, pp. 292-302.

SiBAJA, Rwany; PARrish, Charles (2014), "Pibes, Cracks and Caudillos: Argentina, the World Cup and identity politics", en Soccer \& Society, Vol. 15, No. 5, pp. 655-670.

Violi, Patrizia (1997), Significato ed esperienza, Bompiani, Milano.

Wieviorka, Annette (1998), L’ère du témoin, Plon, Paris. 


\title{
RESUMEN
}

Años después de su retirada del fútbol profesional, Diego Armando Maradona sigue siendo objeto de idolatría. A partir de esta constatación, el presente artículo analiza el discurso argentino sobre el fútbol y su campeón más destacado. De esta forma, se describen las razones por las que la figura de Maradona aparece como un prototipo del así llamado "fútbol criollo" y la encarnación de determinados valores nacionales, características que lo constituyen como ídolo e icono de la cultura popular.

Palabras clave: Maradona, fútbol, héroe, Victor Hugo Morales.

\section{Maradona between Heaven and Earth}

\begin{abstract}
Years after his retirement as a professional football player, Diego Armando Maradona still remains at the centre of idolatric practices. Starting from this consideration, the present article reconstructs the Argentinian discourse on football and describes the reasons for which the figure of Maradona appears to be a prototype of the so called "fútbol criollo" and the embodiment of certain national values, thus leading to his status as an idol and icon of popular culture.
\end{abstract}

Keywords: Maradona, football, hero, Victor Hugo Morales.

\section{RÉSUMÉ}

Beaucoup après sa retraite du football professionnel, Diego Armando Maradona est toujours soumis à des pratiques d'idolâtrie. De là , et analysant ñe discours argentine de football, ce document vise à décrire les raisons pour lesquelles la figure de Maradona apparait comme un prototype du soi-disant « football criollo » que incorpore la réalisation de certaines valeurs nationales, des fonctionnalités qui permettent l'établir comme une idole et une icône de la culture populaire .

Mots-clé: Maradona, héros du football, Victor Hugo Morales. 